\title{
Reflets
}

Revue d'intervention sociale et communautaire

\section{La santé des francophones en situation minoritaire au coeur de nos actions}

\author{
Dominique Cardinal, Caroline Borris, Bernard Pinet et Flavie Leclair
}

Volume 24, numéro 2, automne 2018

URI : https://id.erudit.org/iderudit/1053870ar

DOI : https://doi.org/10.7202/1053870ar

Aller au sommaire du numéro

Éditeur(s)

Reflets, Revue d'intervention sociale et communautaire

ISSN

1203-4576 (imprimé)

1712-8498 (numérique)

Découvrir la revue

Citer cet article

Cardinal, D., Borris, C., Pinet, B. \& Leclair, F. (2018). La santé des francophones en situation minoritaire au coeur de nos actions. Reflets, 24(2), 250-258.

https://doi.org/10.7202/1053870ar

\section{Résumé de l'article}

Au Canada, plus d'un million de francophones habitent à l'extérieur du Québec, parmi lesquels $35 \%$ rapportent avoir peu ou aucunement accès à des services de santé dans leur langue (Gagnon-Arpin, et collab., 2014). En vue de contrer les défis de la santé dans les communautés francophones en situation minoritaire (CFSM), le Consortium national de formation en santé - Volet Université d'Ottawa a développé plusieurs pratiques prometteuses en matière de soutien aux futurs étudiants et étudiantes, à la population étudiante inscrite aux programmes ciblés par le CNFS ainsi qu'aux professionnelles et professionnels, chercheuses et chercheurs du domaine de la santé. Divers programmes sont mis à profit pour soutenir la génération et la diffusion des connaissances. Ces initiatives ainsi que leurs retombées positives méritent l'attention de tous les partenaires qui ont à coeur la santé des francophones.
Tous droits réservés (C) Reflets, Revue d’intervention sociale et communautaire, 2018
Ce document est protégé par la loi sur le droit d'auteur. L'utilisation des services d'Érudit (y compris la reproduction) est assujettie à sa politique d'utilisation que vous pouvez consulter en ligne. 


\title{
La santé des francophones en situation minoritaire au cœur de nos actions
}

\author{
Dominique Cardinal, PHT, M.A. \\ Gestionnaire du volet formation, \\ Consortium national de formation en santé - Volet Université d'Ottawa \\ Caroline Borris, Erg. Aut., M. Éd. \\ Coordonnatrice de projets de formation, \\ Consortium national de formation en santé - Volet Université d'Ottawa \\ Bernard Pinet, M. Sc. \\ Coordonnateur des projets de formation, axe recherche, \\ Consortium national de formation en santé - Volet Université d'Ottawa
}

Flavie Leclair, M.A. (candidate)

Commis à la coordination de projets de formation,

Consortium national de formation en santé - Volet Université d'Ottawa

\section{Résumé}

Au Canada, plus d'un million de francophones habitent à l'extérieur du Québec, parmi lesquels $35 \%$ rapportent avoir peu ou aucunement accès à des services de santé dans leur langue (Gagnon-Arpin, et collab., 2014). En vue de contrer les défis de la santé dans les communautés francophones en situation minoritaire (CFSM), le Consortium national de formation en santé - Volet Université d'Ottawa a développé plusieurs pratiques prometteuses en matière de soutien aux futurs étudiants et étudiantes, à la population étudiante inscrite aux programmes ciblés par le CNFS ainsi qu'aux professionnelles et professionnels, chercheuses et chercheurs du domaine de la santé. Divers programmes sont mis à profit pour soutenir la génération et la diffusion des connaissances. Ces initiatives ainsi que leurs retombées positives méritent l'attention de tous les partenaires qui ont à cœur la santé des francophones. 


\section{Introduction}

Le Canada compte plus d'un million de francophones habitant à l'extérieur du Québec, soit plus de $3 \%$ de la population canadienne (Statistique Canada, 2017). Ils vivent dans un contexte bien particulier, puisqu'ils parlent une langue officielle du pays dans des milieux où l'accès à des services en français peut s'avérer ardu. Par exemple, $35 \%$ des francophones hors Québec estiment qu'il leur serait très difficile de recevoir des services de santé dans leur langue, bien que l'offre de soins en français soit cruciale pour obtenir un service de qualité et sécuritaire (Bouchard et Desmeules, 2011; Gagnon-Arpin, et collab., 2014). En fait, à peine $41 \%$ et $51 \%$ des communautés francophones vivant en situation minoritaire (CFSM) affirment avoir respectivement communiqué en français avec leur médecin et avec le personnel infirmier (Bouchard et Desmeules, 2011). Ces données s'expliquent en partie par de nombreuses lacunes au niveau de l'offre active de services de santé en français auprès des CFSM (Lortie, et collab., 2012). Ces lacunes sont entre autres associées à une pénurie d'effectifs francophones ou bilingues appelés à servir les CFSM en matière de santé (de Moissac, 2016). De plus, le personnel professionnel fait face à des défis importants, notamment l'accès limité aux activités de perfectionnement en français ou encore le manque de ressources humaines et cliniques qui affectent la capacité d'offrir des services en français.

Partie prenante d'un réseau postsecondaire élargi de formation et de recherche en français, le Consortium national de formation en santé (CNFS) - Volet Université d'Ottawa a développé plusieurs initiatives en vue d'améliorer les services de santé en français destinés aux CFSM. Le présent article étale les diverses actions du CNFS en recrutement, en formation et en recherche qui ont été mises en œuvre dans le but de répondre aux défis auxquels les CFSM sont confrontées en matière de santé et de services sociaux. Les partenaires pourront s'appuyer sur ces pratiques prometteuses et leurs retombées pour élaborer des projets similaires visant le même but.

\section{Faire découvrir les programmes en santé aux jeunes francophones}

Le CNFS entreprend chaque année une tournée pancanadienne des écoles secondaires francophones et organise plus d'une dizaine de foires afin de faire découvrir aux jeunes les programmes de première ligne en santé ciblés par le CNFS et de favoriser un choix 
de carrière éclairé. Ces programmes comprennent les suivants : audiologie, ergothérapie, médecine, nutrition, orthophonie, physiothérapie, psychologie, sciences infirmières et service social. Depuis 2003, la Journée des carrières en santé, organisée conjointement par le CNFS et ses partenaires, donne aux jeunes un aperçu pratique des carrières en santé grâce à des ateliers animés par de futurs professionnels et professionnelles. D'ailleurs, l'évaluation de cette activité effectuée en 2016 indique que plus de $75 \%$ des personnes y ayant participé entre 2012 et 2014 ont poursuivi leurs études de premier ou de deuxième cycle dans le domaine de la santé. De plus, le CNFS organise depuis 2008 des présentations interactives par vidéoconférence pour la population étudiante du premier cycle sur les programmes de sciences de la santé de deuxième cycle offerts en français à l'Université d'Ottawa.

Ces activités sont essentielles pour recruter de bons candidats et de bonnes candidates francophones et francophiles qui se dirigent souvent vers des universités québécoises. Elles constituent une occasion de démontrer aux futurs étudiants et étudiantes l'avantage d'étudier dans un milieu bilingue axé sur l'offre active de services.

\section{Soutenir la population étudiante durant la formation universitaire}

Afin de favoriser l'intégration des étudiantes et étudiants de première année en provenance des CFSM à leur nouveau milieu, le CNFS a lancé en 2015 le Projet Ambassadeur. Jumelant un étudiant ou une étudiante de deuxième année à un ou une néophyte, le projet permet aux personnes nouvellement arrivées de s'intégrer rapidement à un réseau de personnes qui vivent des expériences similaires et partagent des intérêts communs, tout en prenant connaissance des diverses activités du CNFS. Selon les évaluations des éditions 2016 et 2017, plus de $75 \%$ des membres de ces cohortes ont trouvé que le projet aidait à renforcer leur sentiment d'appartenance envers la francophonie. Il est connu que ce sentiment de fierté peut inciter les étudiantes et étudiants à poser leur candidature à des postes en région éloignée, favorisant ainsi le recrutement de personnel bilingue (de Moissac, 2016).

Malgré les initiatives mises sur pied pour montrer la nécessité et l'importance d'intervenir auprès des CFSM, convaincre la clientèle étudiante d'effectuer un stage en région rurale demeure un défi. En ce sens, le CNFS a créé le Répertoire des stages en santé en français, qui présente les milieux de stages et dresse un portrait de la situation géographique, sociodémographique et culturelle ainsi que des réalités liées à la santé de 
diverses CFSM situées en régions éloignées. Plus de 80 étudiantes et étudiants se voient aussi octroyer annuellement une aide financière pour effectuer un stage dans les CFSM. D’ailleurs, un sondage effectué en 2016 auprès des personnes diplômées révélait que 74\% des stagiaires sondés étaient retournés travailler dans leur province d'origine.

Des formations ont également été développées afin d'améliorer les habiletés professionnelles de la cohorte CNFS. En 2009, le CNFS a conçu un atelier en ligne, intitulé Cheminer vers la réussite de mon stage, visant à informer les stagiaires des diverses facettes de leur rôle et à les doter de stratégies pour réussir leur stage. Près de 250 étudiantes et étudiants suivent la formation annuellement. Des évaluations réalisées par une consultante externe en 2013 et à l'interne en 2015 concluent que la formation favorise la gestion du stress, la rédaction de bonnes notes cliniques et l'adoption de comportements professionnels. D’ailleurs, plusieurs programmes de l'École de réadaptation de l'Université d'Ottawa l'ont rendue obligatoire dans le cadre de leurs curriculums.

La formation Passe la balle, quant à elle, expose les finissantes et finissants aux notions de base de la supervision dans le milieu de la santé et favorise ainsi la relève. Plus de 120 étudiantes et étudiants participent annuellement à cette formation. De plus, le Projet Antidote a été mis sur pied en 2014 pour soutenir le développement des habiletés linguistiques de la population étudiante grâce à une formation sur l'utilisation du logiciel Antidote. Une cinquantaine de licences sont ainsi distribuées chaque année. La majorité de celles et ceux qui ont participé au projet estiment que celui-ci leur a permis de rehausser la qualité de rédaction de leurs travaux.

Les avantages dont bénéficient les étudiantes et étudiants CNFS sont appréciés. Toutefois, le CNFS constate qu'elles gagneraient à être bonifiées et que d'autres mesures devront se greffer à celles qui sont existantes pour atteindre cet objectif.

\section{Appuyer les professionnelles et professionnels de la santé dans leur formation continue}

Pour répondre aux besoins de formation continue du personnel professionnel pouvant offrir des stages en français, le CNFS propose une panoplie de projets. En 2016, une courte animation en ligne, Être superviseur : un plus à ma vie professionnelle, a été conçue pour conscientiser ces personnes à l'importance de leur rôle dans la formation clinique, les sensibiliser aux bénéfices d'encadrer des stagiaires et leur suggérer des stratégies pour 
relever certains défis liés à la supervision. Un sondage en ligne révélait que les arguments présentés incitaient l'ensemble des participantes et participants à superviser des stagiaires.

Les intervenantes et intervenants ont aussi accès gratuitement à deux séries d'ateliers, présentées en ligne, en présentiel ou par webinaire, destinées à parfaire leurs compétences en supervision. La première série de L'Art de superviser les stagiaires est composée de cinq formations de base et de six formations avancées. Elle présente les notions théoriques et pratiques inhérentes à la supervision en stage. En 2016, le CNFS a traduit en anglais les formations de base afin de soutenir le personnel anglophone qui accueille des stagiaires pouvant effectuer une offre active de services en français. La seconde série regroupe quatre ateliers exposant une problématique spécifique dans le domaine de la santé, mais s'attarde principalement aux troubles de santé mentale, dont l'épuisement professionnel et les troubles neurocognitifs. Suscitant chaque année l'intérêt de près de 900 personnes, l'ensemble des ateliers génère des retombées très positives. En 2013, une étude révélait d'ailleurs que ces formations permettaient aux superviseures et superviseurs de se sentir plus à l'aise avec le processus et d'intégrer des outils et des stratégies d'encadrement dans leurs tâches de supervision (Cardinal, et collab., 2014).

Le CNFS organise plusieurs types de conférences abordant des sujets d'intérêt en santé tels que la santé mentale, la communication et la nutrition. Ces activités sont présentées en français à un auditoire en salle et à distance. Tenues pour la première fois en 2006, les conférences nationales ont permis d'améliorer les connaissances de plus de 1200 personnes réparties dans plus d'une vingtaine de sites au Canada. Elles visent à améliorer les stratégies de prévention et de promotion de la santé dans les CFSM.

Par ailleurs, les conférences-midi du Programme d'excellence professionnelle (PEP) sont un autre moyen qui permet aux intervenantes et intervenants des CFSM de tenir leurs connaissances à jour. Nos données indiquent qu'entre 2013 et 2017, les conférences ont été offertes à plus de 2200 personnes d'un bout à l'autre du Canada et qu'elles répondent à un besoin réel en matière de formation d'appoint en français dans les CFSM. Des sujets tels que la violence envers les femmes, la sexualité, la santé mentale et les soins de longue durée suscitent un vif intérêt auprès des membres de l'auditoire qui ont peu d'occasions $\mathrm{d}$ 'approfondir leur réflexion sur ces thèmes très pertinents.

Le CNFS soutient également les professionnelles et professionnels de la santé qui souhaitent s'engager dans leur communauté, notamment auprès des personnes âgées. À la suite d'une formation par vidéoconférence, ils peuvent animer le programme de prévention des chutes Marche vers le futur. Ce programme vise à réduire les facteurs de risque de chute des personnes âgées grâce à son volet éducatif (capsules d'information) 
et à son volet physique (exercices en groupe et à domicile). La rétroaction reçue indique que le niveau de satisfaction des sites hôtes et des communautés participantes est très élevé. Enfin, d'autres produits éducatifs sont aussi présentés sur le site Web du CNFS. Mentionnons notamment les sites Web Évaluation des personnes âgées et Atteintes et pathologies vécues fréquemment par les personnes suivies en réadaptation, qui répertorient le matériel et les outils en français. Ces derniers contribuent à outiller la communauté professionnelle et à la sensibiliser aux divers aspects touchant la personne, autant les aspects cognitifs que physiques.

\section{Soutenir les capacités de recherche sur la problématique de la santé des CFSM}

De façon complémentaire à la formation, le CNFS contribue au développement de la recherche en français sur la santé des CFSM en soutenant le corps professoral et la population étudiante par l'entremise de divers programmes. Le CNFS a d'ailleurs appuyé 72 projets et octroyé 161 bourses à la communauté étudiante entre 2003 et 2013 pour diverses activités de recherche.

Les principales visées du soutien à la recherche sont de promouvoir les programmes d'appui au corps professoral et à la population étudiante, de faciliter le réseautage entre les acteurs concernés par la recherche, de favoriser la mobilisation des connaissances ainsi que de partager les pratiques prometteuses et les leçons apprises avec les partenaires du CNFS. Dans l'optique de mobiliser les connaissances sur la santé des CFSM, les demandes de soutien de projets doivent couvrir au moins l'une des thématiques prioritaires suivantes: 1) Portraits et déterminants de la santé; 2) Gouvernance et gestion des services de santé; 3) Liens entre la langue, la culture et la santé; 4) Formation, recrutement, maintien en poste et perfectionnement des professionnels de la santé conformément aux exigences en matière de services de santé des CFSM; 5) Accès, offre et prestation de services de santé en français.

Le soutien au corps professoral se décline en deux programmes principaux, soit le démarrage de projets de recherche et le développement d'outils cliniques. Ces derniers visent à accentuer la disponibilité d'outils cliniques en français pour le personnel professionnel en santé et à améliorer l'offre active de services dans cette langue. Le CNFS offre aussi du soutien financier sur une base discrétionnaire en fonction des besoins prioritaires. Des projets porteurs sur l'offre active de services de santé en français et sur la formation 
par simulation interprofessionnelle ont d'ailleurs été soutenus par cette voie. Comme l'indique le tableau 1, un total de 41 projets sur 65 demandes reçues ont obtenu l'appui du CNFS entre 2014 et 2017, soit un taux d'acceptation de près de $65 \%$.

Tableau 1 - Nombre de demandes reçues et de projets soutenus en recherche au CNFS Volet Université d'Ottawa sur une base annuelle lors de la phase IV (2014-2018). Données préliminaires.

\begin{tabular}{|l|c|c|c|c|c|c|c|c|}
\hline & \multicolumn{2}{|c|}{ 2014-2015 } & \multicolumn{2}{c|}{ 2015-2016 } & \multicolumn{2}{c|}{ 2016-2017 } & \multicolumn{2}{c|}{ Total } \\
\hline $\begin{array}{l}\text { Programme de } \\
\text { soutien }\end{array}$ & $\begin{array}{c}\text { Demandes } \\
\text { reçues }\end{array}$ & $\begin{array}{c}\text { Projets } \\
\text { soutenus }\end{array}$ & $\begin{array}{c}\text { Demandes } \\
\text { reçues }\end{array}$ & $\begin{array}{c}\text { Projets } \\
\text { soutenus }\end{array}$ & $\begin{array}{c}\text { Demandes } \\
\text { reçues }\end{array}$ & $\begin{array}{c}\text { Projets } \\
\text { soutenus }\end{array}$ & $\begin{array}{c}\text { Demandes } \\
\text { reçues }\end{array}$ & $\begin{array}{c}\text { Projets } \\
\text { soutenus }\end{array}$ \\
\hline $\begin{array}{l}\text { Corps } \\
\text { professoral }\end{array}$ & 20 & 13 & 16 & 12 & 29 & 16 & 65 & 41 \\
\hline $\begin{array}{l}\text { Population } \\
\text { étudiante }\end{array}$ & 45 & 17 & 48 & 17 & 56 & 21 & 149 & 55 \\
\hline Réseautage & 11 & 11 & 8 & 8 & 7 & 6 & 26 & 25 \\
\hline
\end{tabular}

Le CNFS appuie la population étudiante en recherche par l'entremise des programmes de bourses de recherche, de dissertation et d'été pour initiation à la recherche. Ces programmes contribuent à promouvoir l'intérêt des étudiantes et étudiants envers la problématique de la santé auprès des CFSM, tant à l'intérieur de leur mémoire ou de leur thèse que durant leur formation professionnelle. Le programme de bourses d'été pour initiation à la recherche exerce une double fonction en appuyant également les membres du corps professoral dans leurs programmes de recherche. Le soutien à la communauté étudiante jouit d'une grande popularité comme en témoignent les 149 demandes reçues entre 2014 et 2017. Parmi celles-ci, 55 projets étudiants ont reçu l'appui du CNFS, soit un taux d'acceptation de $37 \%$ (Tableau 1).

Le programme de soutien aux activités de réseautage permet à la communauté universitaire de mobiliser les nouveaux savoirs ayant trait à la santé des CFSM lors d'événements scientifiques, interprofessionnels ou communautaires partout au Canada. Le CNFS a appuyé 25 demandes en ce sens depuis 2014 (Tableau 1). D'ailleurs, une recherche par auteur sur Google Scholar couvrant la même période a permis de recenser 21 articles scientifiques révisés par les pairs où le soutien du CNFS était reconnu. Fait à noter, $76 \%$ de ces publications sont rédigées en français, ce qui favorise la mobilisation des connaissances auprès des CFSM. 
En plus des partenaires universitaires et collégiaux, le CNFS a établi plusieurs partenariats en recherche, notamment avec le Groupe de recherche sur la formation professionnelle en santé et service social en contexte francophone minoritaire (GReFoPS) et l'Institut du savoir Montfort (ISM). Contributeur à la plateforme sur l'approche de recherche collaborative de l'ISM, le CNFS a participé à la mise en place d'une bourse postdoctorale ISM-CNFS en éducation axée sur l'offre active de services de santé en français au Canada.

\section{Conclusion}

En somme, le CNFS - Volet Université d'Ottawa met sur pied et coordonne plusieurs activités favorisant le recrutement et le développement professionnel d'intervenantes et intervenants capables d'offrir des soins de santé de qualité en français. L'accompagnement qu'il offre à la population étudiante ainsi que ses activités de formation offertes en français sont bien documentées dans différents sondages et rapports d'évaluation. Le soutien du CNFS s'étend également aux chercheuses et chercheurs, et le nombre élevé de demandes reçues par l'entremise des divers programmes de recherche témoigne de l'intérêt envers la santé des CFSM. Les initiatives présentées illustrent des pratiques prometteuses ayant généré des retombées positives quant au transfert des connaissances en français et à la formation d'une relève compétente pouvant améliorer les services offerts aux CFSM dans tout le Canada.

\section{Bibliographie}

BOUCHARD, Louise, et Martin DESMEULES (2011). Minorités de langue officielle du Canada: Égales devant la santé?, Québec, Presses de l'Université du Québec, 118 p.

CARDINAL, Dominique, et collab. (2014). "La supervision de stagiaires : un art qui s'apprend", Reflets : revue dintervention sociale et communautaire, Vol. 20, № 1, p. $42-75$.

CONSORTIUM NATIONAL DE FORMATION EN SANTÉ — VOLET UNIVERSITÉ D'OTTAWA (2016). Évaluation des retombées de la Journée des carrières en santé - Rapport final, Ottawa, Consortium national de formation en santé, $89 \mathrm{p}$.

DE MOISSAC, Denis (2016). Accès aux services de santé et d'interprète-accompagnateur : l'expérience des communautés minoritaires à faible densité de francophones au Canada, [rapport de recherche], Winnipeg, Société Santé en français, 75 p. 
GAGNON-ARPIN, Isabelle, et collab. (2014). « Accès et utilisation des services de santé en langue minoritaire », dans Rodrigue Landry (dir.), La vie dans une langue offcielle minoritaire au Canada, Québec, Presses de l'Université Laval, p. 195-223.

LORTIE, Lise, et André J. LALONDE, avec la collaboration de Pier BOUCHARD (2012). Cadre de référence pour la formation à l'offre active des services de santé en français, Ottawa, Consortium national de formation en santé, $25 \mathrm{p}$.

STATISTIQUE CANADA (2017). Proportion de la population selon la langue maternelle déclarée, pour différentes régions au Canada, Recensement de 2016, réf. du 12 octobre 2017, http://www12.statcan.gc.ca/census-recensement/2016/dp-pd/dv-vd/lang/index-fra.cfm 